\title{
MODELO EXPLICATIVO DE FACTORES PSICOLÓGICOS Y EL BULLYING: PRIMER PROYECTO SEMILLERO DE INVESTIGACIÓN DE LA DIRECCIÓN UNIVERSITARIA DE EDUCACIÓN A DISTANCIA DE LA UNIVERSIDAD ALAS PERUANAS \\ Explanatory model of psychological factors and bullying: First Seed Research proyect of the Dirección Universitaria de Educación a Distancia de la Universidad Alas Peruanas
}

\author{
Cleofé Genoveva Alvites-Huamaní ${ }^{1}$ \\ Universidad Alas Peruanas, Perú
}

Recibido: $25-9-2015$

Aceptado: 5-12-2015

\section{RESUMEN}

Esta investigación aborda el tema del bullying como un problema social que afecta al país en diferentes contextos socioeconómicos y demográficos, siendo el objetivo general el analizar las múltiples relaciones entre la depresión, ansiedad, autoestima, riesgo suicida, clima familiar y autoconcepto como factores psicológicos relacionados con el bullying en estudiantes de 4to y 5 to grado de primaria de instituciones públicas de Lima (San Juan de Lurigancho), Arequipa (Cerro Azul) y Trujillo (El Porvenir). Es un estudio de alcance explicativo y diseño no experimental, transeccional correlacional-causal, que buscó predecir el comportamiento de una variable (bullying) a partir de otras (depresión, ansiedad, autoestima, riesgo suicida, clima familiar y autoconcepto), consideradas como variables predictoras, para lo cual se aplicó la regresión múltiple, en una población de 3000 estudiantes de 4to y 5 to grado de educación primaria de 16 instituciones educativas y una muestra de 1730 sujetos. Los instrumentos utilizados para medir las variables son: el Cuestionario de Depresión Infantil (CDI) de Kovacs, de 27 ítems;, la Escala de Ansiedad para niños de Spence (SCAS), de 38 ítems; la Escala de Autoestima de Coopersmith Escolar (SEI), de 50 ítems y la Escala de Riesgo Suicida (ERS), de 36 ítems; la escala de Clima Familiar (FES), de 90 ítems; la Escala de Autoconcepto de Piers-Harris, de 80 ítems y el Auto-test de Cisneros: Acoso Escolar, que mide el Bullying con 50 ítems. Los resultados demostraron que están relacionados la depresión, ansiedad, riesgo suicida y bullying (indice global de acoso) en los estudiantes de cuarto y quinto grado, siendo la ansiedad ( $p$ $=.000$ ) la variable que explica mejor la incidencia en el Bullying (indice global de acoso) en ambos grados, seguido de la depresión ( $p=.015$, en 4 to grado y $p=.000$ en 5to grado).

Palabras Clave: Depresión, ansiedad, riesgo suicida y bullying, indice global de acoso, clima familiar, autoconcepto, factores psicológicos.

1 Directora, asesora y revisora de Tesis de la Unidad de Investigación de Post Grado de diversas universidades, docente-tutor de la Universidad Virtual de Michoacan, México. Coordinadora de la División de Investigación y Extensión Científica Tecnológica de la Direccion Universitaria de Educación a Distancia de la Universidad Alas Peruanas, adscrita al Directorio Nacional de Investigadores e Innovadores y miembro del programa Mujeres Científicas. cleoalvitesh@gmail.com; c_alvites@uap.edu.pe 


\section{Abstract}

This research addresses the issue of bullying as a social problem affecting the country in different socioeconomic and demographic contexts. The main objective of this study is to analyze the multiple relationships among depression, anxiety, self-esteem, suicidal risk, family atmosphere and self-concept as psychological factors related to bullying in primary students from 4th and 5th grade in public schools in Lima (San Juan de Lurigancho), Arequipa (Cerro Azul) and Trujillo (El Porvenir). This is a study with an explanatory scope and a non-experimental design, a cross-sectional, explanatory -correlational research, which attempted to predict the behavior of a variable (bullying) in relation to others (depression, anxiety, self-esteem, suicidal risk, family atmosphere and self-concept), considered as predictor variables. Multiple regressions were applied, in a population of 3,000 primary students from 4th and 5th grade in 16 public schools and a sample of 1730 subjects. The instruments used to measure the variables are: the Kovacs Children's Depression Inventory (CDI) with 27 items; the Spence Children's Anxiety Scale (SCAS) with 38 items; the school Coopersmith Self-Esteem Inventory (SEI) with 50 items and the Suicide Risk Scale (SRS) with 36 items; the Family Atmosphere Scale (FAS) with 90 items; the Piers-Harris Self-Concept Scale with 80 items and the Cisneros Self-Test: Bullying, which measures Bullying with 50 items. The results demonstrated that depression, anxiety, suicidal risk and bullying (global rate of harassment) are related with fourth and fifth graders, with anxiety $(p=.000)$ as the variable that best explains the impact on Bullying ( global rate of harassment) in both grades, followed by depression $(p=.015$, in 4 th grade and $p=$ .000 in 5 th grade).

Keywords: Depression, anxiety, suicidal risk and bullying, global rate of harassment, family atmosphere, self-concept, psychological factors.

\section{INTRODUCCIÓN}

Debemos resaltar que este primer proyecto forma parte del semillero de investigación de la División de Investigación y Extensión Científica Tecnológica de la Dirección Universitaria de Educación a Distancia (DUED) de la Universidad Alas Peruanas, el cual está relacionado con el bullying o acoso escolar en poblaciones vulnerables que registran un mayor índice de violencia, debido a que es un problema social que afecta al país en diferentes contextos socioeconómico y demográficos. Precisamente, según Fernández (2014, febrero 20), el $44 \%$ de los estudiantes de Lima y Callao han sufrido algún tipo de acoso en el colegio y un $14 \%$ de ellos pensó en suicidarse. A la luz de esta problemática, el primer semillero de investigación de la DIECT-DUED-UAP consideró relevante realizar un proyecto enmarcado en el "bullying o acoso escolar", desde la evaluación, diagnóstico y psicoterapia, mediante la aplicación de las tecnologías de la información y comuni- cación. Se tuvo como variables la depresión, ansiedad, autoestima, riesgo suicida, clima social familiar y autoconcepto y su probable influencia en el bullying. Por ello, se planteó como objetivo general analizar las múltiples relaciones entre la depresión, ansiedad, autoestima, riesgo suicida, clima familiar y autoconcepto como factores psicológicos del bullying en estudiantes de 4to y 5 to grado de primaria de instituciones públicas y privadas de Lima (San Juan de Lurigancho), Arequipa (Cerro Azul) y Trujillo (El Porvenir). Como objetivos específicos se proyectó: i) determinar la relación entre depresión, ansiedad, riesgo suicida y bullying; ii) establecer la relación entre la autoestima, clima familiar, autoconcepto y bullying y iii) determinar la relación entre autoestima, riesgo suicida, clima familiar y bullying en los estudiantes del 5 to grado de primaria. Para verificar los objetivos se formularon seis hipótesis: H1: La depresión, ansiedad, riesgo suicida y el indice global de acoso (bullying) están relacionados; $\mathrm{H} 2$ : 
La autoestima, clima familiar, autoconcepto y el indice global de acoso (bullying) están relacionados; H3: El clima familiar, autoestima, riesgo suicida y la intensidad de acoso (bullying) están relacionados; H4: La depresión, ansiedad, riesgo suicida y la intensidad de acoso (bullying) están relacionados; H5: La autoestima, clima familiar, autoconcepto y la intensidad de acoso (bullying) están relacionados; y H6: El clima familiar, autoestima, riesgo suicida y la intensidad de acoso (bullying) están relacionados.

\section{Bullying o acoso escolar en el Perú}

Desde que Dan Olwes (1978) inició las investigaciones sobre el bullying o acoso escolar en Noruega, definiéndolo como una conducta de persecución física y/o psicológica que realiza un estudiante contra otro al que escoge como víctima de repetidos ataques, esta acción negativa e intencionada sitúa a la víctima en una posición de la que difícilmente puede escapar por sus propios medios. Esta acción afecta negativamente la autoestima del individuo, genera estados de ansiedad y depresión en la víctima, dificultando su integración en el medio.

Según RPP Noticias (2014, agosto 30), el bullying se inicia en el hogar; si en este prima la violencia, un estilo de crianza autoritario, la sobreprotección, la falta de comunicación y respeto, se está criando a un niño agresor o a una víctima de bullying. El Comercio (2014, agosto 27) refiere que Lima se ha convertido en la provincia con mayor índice de violencia escolar (70\%), con $1052 \mathrm{ca}$ sos de los 1362 que existen a nivel nacional, entre septiembre 2013 a agosto del 2014. La violencia física es la que predomina, seguida de la violencia psicológica. Según Pichihua (2013, septiembre 10) estudiantes entre los 8 y 17 años deciden suicidarse como consecuencia del bullying y la depresión, siendo la provincia de Lima donde hay mayor prevalencia (70\%), seguida de Arequipa, Huancayo, Cusco y Pucallpa; habiendo sucedido 20 suicidios a nivel nacional. Pese a que en 2012 el $18 \%$ de la población suicida eran menores de edad, en 2013 se redujo a un 10\%, colocando al Perú en el tercer país de América Latina con más suicidios de menores de edad. Adicionalmente, cabe resaltar que la violencia familiar contra los menores de edad tiene una alta incidencia en la provincia de Lima (25\%), siendo los distritos de San Juan de Lurigancho, Villa Maria del Triunfo y Villa El Salvador donde hay mayor incidencia de bullying; incluyendo también a San Juan de Miraflores y Comas (La Republica 2014, septiembre $25)$. Con relación a las provincias del interior del país, Trujillo y Arequipa son las que tienen la mayor incidencia de violencia; destacando el distrito del Porvenir y Cerro Colorado. La información reseñada permite afirmar que el bullying no es un problema aislado de otros aspectos, sino que tiene implícitas una serie de variables que deberían ser consideradas en su evaluación y diagnóstico como la depresión, autoestima, riesgo suicida, clima social familiar, ansiedad, autoconcepto y el bullying en sí mismo.

\section{FACTORES PSICOLÓGICOS Y BULLYING O ACOSO ESCOLAR}

Considerando que las variables depresión, ansiedad, autoestima, riesgo suicida, clima familiar y autoconcepto incluyen criterios psicológicos, estos se han enmarcado dentro de los factores psicológicos, tomando como referencia a Gómez, (2014), quien menciona que existe factores asociados a la violencia, dentro de los cuales se encuentra los factores relacionados a variables psicológicas, siendo la perspectiva patogénica de la salud que se describe en este estudio referidas a depresión, autoestima, autoconcepto y ansiedad, para el clima familiar la teoría cognitiva-social al tener como influencia las consecuencias ambientales y sociales del entorno, en el caso del riesgo suicida está sustentada en la Psicologia social, como un conjunto de expresiones agresivas condicionados por aspectos históricos, sociales y culturales en actuar violentamente contra las personas o contra sí mismo.

Salgado (2012) enfatiza que el bullying puede ocasionar en las víctimas: síntomas depresivos, angustia, fobias sociales, ansiedad, baja autoestima y conductas problemáticas que pueden llegar al suicidio. Por su parte Loredo-Abdalá et al. (2008), indican que tanto víctimas como agreso- 
Modelo explicativo de factores psicológicos y el bullying: Primer proyecto Semillero de Investigación de la Direccion Universitaria de Educación a Distancia de la Universidad Alas Peruanas

res tienen mayor riesgo de sufrir depresión y tener ideas suicidas, ansiedad, baja autoestima y trastorno de sueńo, entre otros síntomas. Oliveros et al. (2008), en su investigación sobre bullying en colegios estatales de primaria en Perú, al analizar el perfil de la víctima en el nivel escolar de primaria refieren que el acoso del que son víctimas les produce sentimientos de tristeza, afecta su autoestima, favorecen la depresión y, en situaciones extremas, hasta el suicidio. La ley que promueve la sana convivencia y disciplina escolar y sanciona el acoso y violencia entre escolares (Ley antibullying en Perú). Esta norma resalta que la situación de acoso genera en la persona que la padece: miedo, tristeza, baja autoestima, lo cual influye negativamente en su vida presente y futura; dificulta sus relaciones sociales, afecta la seguridad y confianza en sí mismo. Arroyave (2012), al referirse a factores de vulnerabilidad y riesgo asociados al bullying, indica que las víctimas son el grupo con mayor patología mental. Presentan en mayor porcentaje depresión, ansiedad y trastorno por déficit de atención con predominio impulsivo, baja autoestima, inseguridad y poco asertividad. Las consecuencias más impactantes son la ideación e intento de suicidio que se presenta tanto en víctimas como en acosadores; los pensamientos suicidas son los de mayor prevalencia en mujeres que en hombres. Generalmente, cuando son evaluados, el diagnóstico es fobia social, depresión y ansiedad. Leganes (2013), en su propuesta de intervención para prevenir el acoso escolar desde una perspectiva sociogrupal, describe que el bullying o el maltrato entre iguales es un tipo de comportamiento violento y reiterado sobre una misma persona. La continuidad del maltrato genera aspectos negativos de ansiedad, descenso de la autoestima, cuadros depresivos que dificultan su integración en el medio escolar y el desarrollo normal de los aprendizajes e incide en su desarrollo social, psicológico, biológico y afectivo. Según Oliveros et al. (2009), la intimidación tiene origen multicausual, ocasiona problemas en la salud física, emocional, social; produce fobia y ansiedad al hacerles sentirse perseguidos e intimidados por algunos de sus compañeros. Collell y Escudé (2006) manifiestan que todos los estudiantes implicados en situaciones de maltrato sean víctimas o agresores están en mayor situación de trastornos psicopatológicos en la adolescencia o en la vida adulta. Fernández (2014, febrero 20) afirma que el $44 \%$ de estudiantes de Lima y Callao han sufrido acoso escolar y el $14 \%$ pensó en suicidarse.

\section{Método}

Participantes. Debido a que el bullying es un problema social de cobertura nacional, pero, según estadísticas, con más casos de violencia en determinados distritos de departamento, se eligió como población a estudiantes de zonas vulnerables ubicadas en tres departamentos del Perú: San Juan de Lurigancho del departamento de Lima (6 instituciones educativas); Cerro Colorado, del Departamento de Arequipa (5 instituciones educativas) y distrito del Porvenir, de la provincia de Trujillo (5 instituciones educativas). Las edades de los participantes oscilaron entre los 8 y 13 ańos. Los 3000 sujetos (de ambos géneros) comprendidos en el estudio provenían de diversos estratos sociales.

Para la selección de la muestra se consideró como criterios de inclusión la participación voluntaria de las instituciones educativas de los distritos elegidos, de las aulas del 4to y 5 to grado de educación primaria. Se incluyeron 2 instituciones educativas de San Juan de Lurigancho (Lima), 4 de Cerro Colorado (Arequipa) y 3 de El Porvenir (Trujillo); en total: 9 instituciones educativas. La muestra, de tipo no probabilística, incluyó a 1730 estudiantes.

Instrumentos. Para recolectar los datos se utilizaron 7 instrumentos, que midieron los 6 factores psicológicos y 1 instrumento para evaluar el bullying en sí mismo.

Para evaluar la depresión se utilizó el Cuestionario de Depresión Infantil (CDI) de Kovacs, que valora síntomas depresivos esenciales en niños y adolescentes de 7 a 17 años, en dos factores: estado de ánimo disfórico y autoestima negativa. El cuestionario consta de 27 ítems, que permiten obtener información acerca del nivel de depresión total, así como de los dos factores mencionados. Este instrumento fue validado en población peruana con un nivel de confiabilidad de 0.84 . 
En la medición de la ansiedad se utilizó la Escala de Ansiedad para niños de Spence (SCAS), que consta de 6 factores que evalúan pánico-agorafobia, ansiedad por separación, fobia social, miedo al daño físico, trastorno obsesivo compulsivo y ansiedad generalizada. La escala consta de 38 ítems; su nivel de confiabilidad es 0.88 ; se aplica a estudiantes de 8 a 12 años y sirve para medir los trastornos de ansiedad en la niñez y adolescencia.

Para valorar la variable autoestima se empleó la Escala de Autoestima de Coopersmith Escolar (SEI), que evalúa la autoestima general y 4 facto- res (si mismo general, social-pares, hogar-padres y escuela) para estudiantes de 8 a 15 años. Esta escala consta de 50 ítems sobre niveles de autoestima, con 8 ítems de escala de mentiras. Si la respuesta a estos es positiva se invalida la prueba. La confiabilidad del instrumento fue de 0.90.

Para estimar el riesgo suicida se aplicó la Escala de Riesgo Suicida (ERS), que consta de 4 factores: conducta suicida, intento suicida, satisfacción, afectos negativos y sentido de vida. Posee 36 ítems y su confiabilidad en población peruana fue de 0.90 . Se aplica a estudiantes de 8 a 13 años.

Tabla 1. Descripción de los 6 instrumentos utilizados para la recolección de datos de la variable Síntomas psicosomáticos

\begin{tabular}{|c|c|c|c|}
\hline Factores & Indicador & Ítem & Instrumento \\
\hline \multirow{2}{*}{ Depresión } & Disforia & $2,3,6,11,13,14,15,19,23,24,26$ & \multirow{2}{*}{ CDI } \\
\hline & Autoestima negativa & $1,4,5,7,8,9,10,12,16,17,18,20,21,22,25,27$ & \\
\hline \multirow{6}{*}{ Ansiedad } & Ansiedad por separación & $5,8,11,14,15,38$ & \multirow{6}{*}{ SCAS } \\
\hline & Pánico-agorafobia & $12,19,25,27,28,30,32,33,34$ & \\
\hline & Fobia social & $6,7,9,10,26,31$ & \\
\hline & Miedo al daño físico & $2,16,21,23,29$ & \\
\hline & Trastorno obsesivo-compulsivo & $13,17,24,35,36,37$ & \\
\hline & Ansiedad generalizada & $1,3,4,18,20,22$ & \\
\hline \multirow{5}{*}{ Autoestima } & Si mismo & $\begin{array}{l}1,3,4,7,10,12,13,15,18,19,24,25,27,30,31 \\
34,35,38,39,43,47,48,51,55,56,57\end{array}$ & \multirow{5}{*}{ SEl } \\
\hline & Social-pares & $5,8,14,21,28,40,49,52$ & \\
\hline & Hogar-padre & $6,9,11,16,20,22,29,44$ & \\
\hline & Escuela & $2,17,23,33,37,42,46,54$ & \\
\hline & Mentiras & $26,32,36,41,45,50,53,58$ & \\
\hline \multirow{4}{*}{ Riesgo suicida } & Conducta Suicida & $\begin{array}{l}2,14,16,15,17,4,3,11,12,13,6,1,10,7,8,5 \text {, } \\
9,36\end{array}$ & \multirow{4}{*}{ ERS } \\
\hline & Satisfacción & $19,20,18,22,21,23,24,25$ & \\
\hline & Afectos negativos & $32,34,35,31,33$ & \\
\hline & Sentido de Vida & $26,27,28,29,30$ & \\
\hline \multirow{3}{*}{$\begin{array}{l}\text { Clima } \\
\text { familiar }\end{array}$} & Relaciones & $\begin{array}{l}1,11,21,31,41,51,61,71,81,2,12,22,32,42,52 \\
62,72,82,3,13,23,33,43,53,63,73,83\end{array}$ & \multirow{3}{*}{ FES } \\
\hline & Desarrollo & $\begin{array}{l}4,14,24,34,44,54,64,74,84,5,15,25,35,45,55 \\
65,75,85,6,16,26,36,46,56,66,76,86,7,17,27 \\
37,47,57,67,77,87,8,18,28,38,48,58,68,78,88\end{array}$ & \\
\hline & Estabilidad & $\begin{array}{l}9,19,29,39,49,59,69,79,89,10,20,30,40,50 \\
60,70,80,90\end{array}$ & \\
\hline \multirow{6}{*}{ Autoconcepto } & Conductual & $\begin{array}{l}13,14,18,22,25,31,32,34,35,45,48,56,67,72 \\
75,76,78,80\end{array}$ & \multirow{6}{*}{ TAC } \\
\hline & Intelectual & $\begin{array}{l}5,9,12,16,17,21,23,24,26,27,30,33,42,49,53 \\
66,70\end{array}$ & \\
\hline & Físico & $8,15,19,29,41,47,54,60,63,64,65,73$ & \\
\hline & Falta de Ansiedad & $6,7,10,20,28,37,44,55,61,68,74,79$ & \\
\hline & Popularidad & $1,3,11,40,46,51,57,58,62,69,71,77$ & \\
\hline & Felicidad-Satisfacción & $2,4,36,38,39,43,50,52,59$ & \\
\hline
\end{tabular}


Modelo explicativo de factores psicológicos y el bullying: Primer proyecto Semillero de Investigación de la Direccion Universitaria de Educación a Distancia de la Universidad Alas Peruanas

Con la variable Clima social Familiar se utilizó la escala de Clima Familiar (FES), que evalúa y describe las relaciones interpersonales entre los miembros de la familia en tres factores: relaciones, desarrollo y estabilidad. Tiene 90 ítems. Obtuvo una confiabilidad de 0.95 en población peruana. Se aplica a un grupo etáreo de 8 a 15 años.

En la medición del autoconcepto se utilizó la Escala de Autoconcepto de Piers-Harris, que evalúa a estudiantes de 7 a 12 años, en 6 factores: autoconcepto conductual, intelectual, físico, falta de ansiedad, popularidad y felicidad-satisfacción. Mide el nivel de autoconcepto global y por fac- tores. Consta de 80 ítems y su confiablidad en población peruana fue de 0.89 .

Para la variable bullying se aplicó el Auto-test de Cisneros: Acoso Escolar, que evalúa a estudiantes entre 7 y 16 ańos. Consta de 50 ítems distribuidos en 8 factores. En población peruana tuvo una confiabilidad de 0.86 .

Los 7 instrumentos descritos se pueden administrar de forma colectiva con una duración de 20 a 30 minutos. En la tabla 1 se describen los instrumentos utilizados.

Tabla 2. Descripción del instrumento utilizado para la recolección de datos de la variable Bullying

\begin{tabular}{|c|c|c|c|}
\hline Factor & Indicador & Ítem & Instrumento \\
\hline \multirow{2}{*}{$\begin{array}{l}\text { Índice Global } \\
\text { de Acoso }\end{array}$} & Desprecio-Ridiculización & $2,3,6,9,19,20,26,27,31,32,33,34,35,36,44,46,50$ & \multirow{8}{*}{ Test Bull } \\
\hline & Coacción & $7,8,11,12,13,14,47,48$ & \\
\hline \multirow{6}{*}{$\begin{array}{l}\text { Intensidad del } \\
\text { acoso }\end{array}$} & Restricción-comunicación & $1,2,4,5,10$ & \\
\hline & Agresiones & $15,19,23,24,28,29,39$ & \\
\hline & Intimidación-amenazas & $28,29,39,40,41,42,43,47,48,49$ & \\
\hline & Exclusión-Bloqueo social & $10,17,18,21,22,31,38,41,45$ & \\
\hline & Hostigamiento Verbal & $3,6,17,19,20,24,25,26,27,30,37,38$ & \\
\hline & Robos & $13,14,15,16$ & \\
\hline
\end{tabular}

\section{Tipo y diseño}

El estudio es de tipo explicativo, diseño no experimental, transeccional correlacional-causal (Hernández, Fernández y Baptista, 2014). Se explica la relación de causalidad entre las variables en un momento determinado. Se pretendió predecir el comportamiento de una variable (bullying) a partir de otras (depresión, ansiedad, autoestima, riesgo suicida, clima familiar y autoconcepto), consideradas como variables predictoras.

\section{Procedimiento}

La ejecución del proyecto "Bullying" consta de cuatro fases.

Primera fase. Modelo explicativo de los factores psicológicos y el bullying. Se evalúo y diagnosticó el bullying en la muestra seleccionada. Para el desarrollo de esta fase, la muestra se subdividió en
6 etapas.

En la etapa 1 (planeación, organización y desarrollo del proyecto) se invitó a estudiantes de las Escuelas Académico Profesionales (EAP) de Psicología Humana e Ingeniería de Sistemas para que participaran del semillero en forma voluntaria.

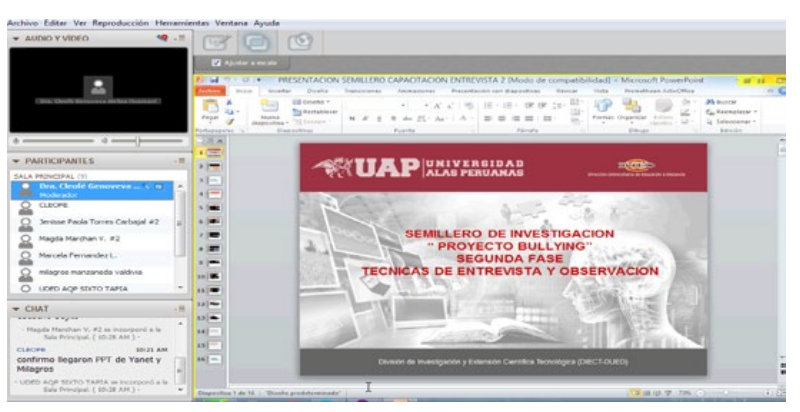

Figura 1. Capacitación a estudiantes del semillero de investigación mediante sala de conferencias. 
En la etapa 2 se realizó la capacitación a las 15 estudiantes de la EAP de Psicologia Humana que conformaban el semillero de las tres UDED (Unidad Descentralizada de Educación a Distancia). Se desarrollaron sesiones para capacitarlas en el manejo de los instrumentos y brindarles pautas para evaluar a los estudiantes. Estas sesiones se ejecutaron de manera sincrónica, una vez por semana, durante dos meses, mediante la plataforma de Blackboard a través de la sala de conferencia Illuminate; tres días de manera presencial, en cada una de las provincias seleccionadas, para que realicen la administración de los 7 instrumentos seleccionados para evaluar y diagnosticar el bullying y los factores psicológicos en los participantes.

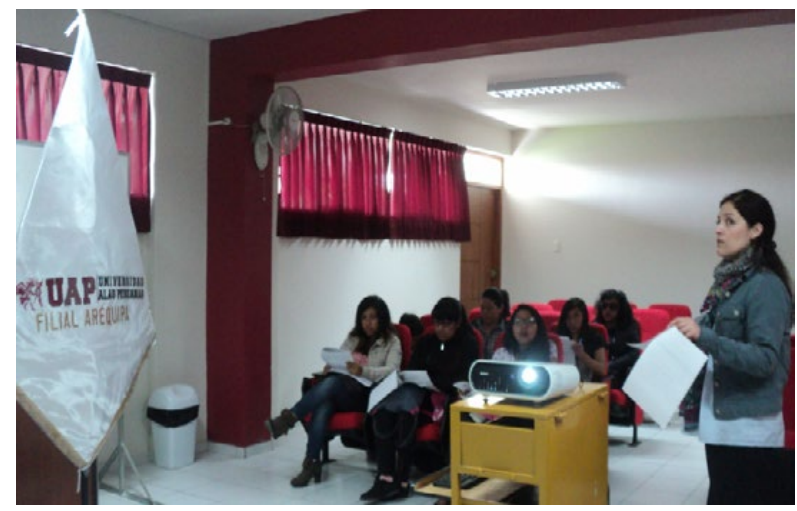

Figura 2. Capacitación a estudiantes del semillero UDED Arequipa

En la etapa 3 se administraron los instrumentos de recolección de datos en los distritos de San Juan de Lurigancho (Lima), Cerro Colorado (Arequipa) y El Porvenir (Trujillo), a razón de una semana por distrito. Previamente se realizaron las coordinaciones con los directores de las instituciones educativas seleccionadas, quienes aceptaron participar en el proyecto. A ellos se les envió una carta de presentación y el consentimiento informado para que sea firmado por los padres de familia de los menores del 4to y 5to grado de primaria. Obtenida la autorización y el consentimiento firmado, se concurrió a las instituciones educativas para evaluar a los estudiantes en el lapso de una semana en cada distrito seleccionado.

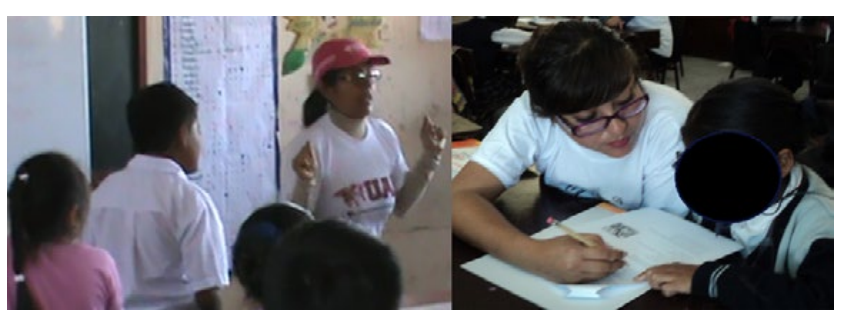

Figura 3. Estudiante Semillero DUED-Lima y UDED Arequipa realizando evaluación.

En la etapa 4 de esta primera fase se realizó el consolidado de resultados de los instrumentos utilizando el programa Excel para la descripción estadística de los resultados. También se elaboraron los informes por grado y por cada colegio participante. Estos informes se entregaron a los directores de las instituciones educativas participantes. Para preservar el derecho de la confidencialidad de los participantes, se omitieron los datos de cada uno de ellos, brindándose el informe general por aula, como se puede observar en la siguiente figura.

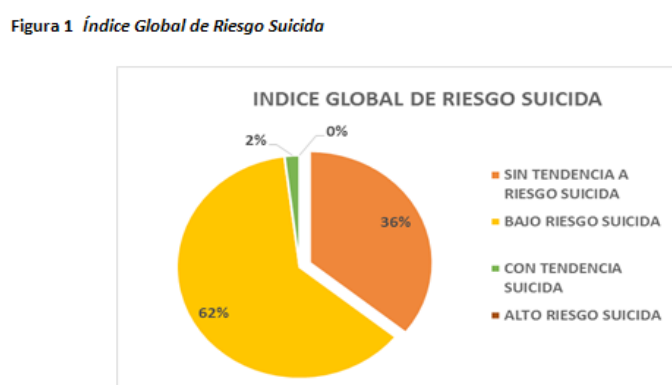

Se puede observar que un $36 \%$ de estudiantes del 5 to de primaria, no presenta RIESGO SUICIDA; un $62 \%$ de los mismos se encuentran en el nivel BAJO RIESGO SUICIDA; el $2 \%$ de ellos presentan un NIVEL CON TENDENCIA SUICIDA y el \%\% a un nivel de ALTO RIESGO SUICIDA.

Concluyendo a nivel general el $2 \%$ de los estudiantes muestran TENDENCIA SUICIDA y ninguno con un ALTO RIESGO SUICIDA.

Figura 4. Parte de informe remitido a las instituciones educativas participantes.

Segunda, tercera y cuarta fases. Se encuentran en desarrollo, pues el proyecto tiene una duración de dos años; se inició en septiembre del 2014. En la segunda fase se realizará el diseño y creación de software de psicoeducación para el tratamiento del Bullying; este será ejecutado por los estudiantes del semillero de investigación de la EAP Ingeniería de Sistemas. En la fase 3 se aplicará el software de psicoeducación en bullying a los estudiantes que fueron evaluados en la primera fase y en quienes se detectó el problema. 
Modelo explicativo de factores psicológicos y el bullying: Primer proyecto Semillero de Investigación de la Direccion Universitaria de Educación a Distancia de la Universidad Alas Peruanas

\section{RESULTADOS}

Para obtener los resultados de la administración de los 7 instrumentos se hizo la contrastación de las hipótesis utilizando el software estadístico SPPS v.19. Mediante regresión lineal múltiple se determinó el grado de asociación entre las diversas supuestas variables explicativas (depresión, ansiedad, autoestima, riesgo suicida, clima social familiar y autoconcepto) como factores psicológicos y una variable dependiente (bullying). Se trató de buscar la variable que mejor explique a la variable dependiente, medida a través del índice de acoso global e intensidad del acoso. Los análisis se realizaron con el total de la muestra, en forma separada, por grado de estudios, tal como se detalla a continuación.

Pruebas de hipótesis para los datos de 4to grado

Con la primera prueba de hipótesis de los estudiantes del 4to grado de primaria de las instituciones educativas de San Juan de Lurigancho (Lima), Cerro Azul (Arequipa) y El Porvenir (Trujillo) se obtuvo que el valor del nivel crítico $(p=.000)$ indica que existe relación lineal significativa. Por lo tanto, se rechazó la hipótesis nula y se concluyó que la depresión, ansiedad, riesgo suicida y el indice global de acoso están relacionados, como se muestra en la tabla 3.

\begin{tabular}{|c|c|c|c|c|c|c|}
\hline \multicolumn{7}{|c|}{ ANOVA $^{3}$} \\
\hline & & $\begin{array}{l}\text { Sum of } \\
\text { Squares }\end{array}$ & df & Mean Square & $\mathbf{F}$ & Sig. \\
\hline \multirow{3}{*}{$\frac{M}{1}$} & Regression & 93,043 & 3 & 31,014 & 20,360 & $.000^{6}$ \\
\hline & Residual & 1239,975 & 814 & 1,523 & & \\
\hline & Total & 1333,018 & 817 & & & \\
\hline
\end{tabular}

a. Dependent Variable: INDICEGLOBALDEACOSO

b. Predictors: (Constant), DEPRESION ANSIEDAD RIESGOSUICIDA,

Tabla 3. Análisis de las variables: depresión, ansiedad, riesgo suicida y el indice global de acoso

De acuerdo con el nivel crítico asociado a cada prueba $t$, se concluyó que 2 de las 3 variables utilizadas poseen coeficientes significativos $(p<.05)$. Por tanto, contribuyen a explicar lo que ocurre con la variable dependiente, excepto la variable riesgo suicida $(p=.967)$, como se muestra en la tabla 4.

\begin{tabular}{|c|c|c|c|c|c|c|}
\hline \multicolumn{7}{|c|}{ Coefficients $^{2}$} \\
\hline \multirow[b]{2}{*}{ Model } & & \multicolumn{2}{|c|}{ Unstandardized Coefficients } & \multirow{2}{*}{$\begin{array}{c}\text { Standardized } \\
\text { Coefficients } \\
\text { Beta }\end{array}$} & \multirow[b]{2}{*}{$t$} & \multirow[b]{2}{*}{ Sig. } \\
\hline & & $B$ & Std. Error & & & \\
\hline 1 & (Constant) & 2,162 & .366 & & 5,911 & .000 \\
\hline & ANSIEDAD & .385 & .063 & 207 & 6,130 & .000 \\
\hline & DEPRESION & ,202 & 083 & 163 & 2,432 & 015 \\
\hline & RIESGOSUICIDA &,- 003 & .069 &,- 003 &,- 041 & 967 \\
\hline
\end{tabular}

Tabla 4. Análisis de los coeficientes de las variables: depresión, ansiedad, riesgo suicida y el indice global de acoso

La segunda prueba de hipótesis de los estudiantes de 4to. Grado de primaria dio $p=.000$ con relación lineal significativa. Por lo tanto, se rechazó la hipótesis nula y se concluyó que la autoestima, clima familiar, autoconcepto y el índice global de acoso están relacionados (tabla 5).

\begin{tabular}{|c|c|c|c|c|c|c|}
\hline \multicolumn{7}{|c|}{ ANOVA $^{2}$} \\
\hline \multicolumn{2}{|l|}{ Model } & $\begin{array}{l}\text { Sum of } \\
\text { Squares }\end{array}$ & df & Mean Square & $\mathrm{F}$ & Sig. \\
\hline \multirow[t]{3}{*}{1} & Regression & 41,729 & 3 & 13,910 & 8,768 &, $000^{\mathrm{b}}$ \\
\hline & Residual & 1291,290 & 814 & 1,586 & & \\
\hline & Total & 1333,018 & 817 & & & \\
\hline
\end{tabular}

b. Predictors: (Constant), AUTOESTIMA CLIMAFAMILIAR AUTOCONCEPTO,

Tabla 5. Análisis de las variables: La autoestima, clima familiar, autoconcepto y el indice global de acoso

De acuerdo con el nivel crítico asociado a cada prueba t, se concluyó que 2 de las 3 variables utilizadas poseen coeficientes significativos $(p<$ $.05)$ y contribuyen a explicar lo que ocurre con la variable dependiente, excepto la variable autoconcepto, cuyo nivel de significancia fue $p=.532$ (tabla 6).

\begin{tabular}{|c|c|c|c|c|c|c|}
\hline \multicolumn{7}{|c|}{ Coefficients $\mathrm{s}^{\mathrm{a}}$} \\
\hline \multirow[b]{2}{*}{ Model } & & \multicolumn{2}{|c|}{ Unstandardized Coefficients } & \multirow{2}{*}{$\begin{array}{c}\text { Standardized } \\
\text { Coefficients }\end{array}$} & \multirow[b]{2}{*}{$t$} & \multirow[b]{2}{*}{ Sig. } \\
\hline & & $B$ & Std. Error & & & \\
\hline 1 & (Constant) & 2,791 & .251 & & 11,140 & .000 \\
\hline & CLIMAFAMILIAR & .269 & .063 & .150 & 4,279 & .000 \\
\hline & AUTOESTIMA &,- 172 & 056 & -107 & $-3,092$ & ,002 \\
\hline & AUTOCONCEPTO &,- 019 & .031 & -.022 & -625 & .532 \\
\hline
\end{tabular}

Tabla 6. Análisis de los coeficientes de las variables: Autoestima, clima familiar, autoconcepto y el indice global de acoso

La tercera prueba de hipótesis de esta muestra de estudiantes de 4to grado de primaria dio $p=.000$, indicando que existe relación lineal significativa. Por lo tanto, se rechazó la hipótesis nula y se concluyó que el clima familiar, autoestima, riesgo suicida y el indice global de acoso están relacionados (tabla 7). 


\begin{tabular}{|c|c|c|c|c|c|c|}
\hline \multicolumn{7}{|c|}{ ANOVA $^{a}$} \\
\hline \multicolumn{2}{|c|}{ Model } & $\begin{array}{l}\text { Sum of } \\
\text { Squares }\end{array}$ & df & Mean Square & $\mathrm{F}$ & Sig. \\
\hline \multirow[t]{3}{*}{1} & Regression & 76,593 & 3 & 25,531 & 16,541 & $000^{b}$ \\
\hline & Residual & 1256,426 & 814 & 1,544 & & \\
\hline & Total & 1333,018 & 817 & & & \\
\hline
\end{tabular}

a. Dependent Variable: INDICEGLOBALDEACOSO

b. Predictors: (Constant), CLIMAFAMILIAR, AUTOESTIMA, RIESGOSUICIDA

Tabla 7. Análisis de las variables: El clima familiar, Autoestima, riesgo suicida y el indice global de acoso

De acuerdo con el nivel crítico asociado a cada prueba t, se concluyó que las 3 variables utilizadas poseen coeficientes significativos $(p<.05)$. Por tanto, todas contribuyen a explicar lo que ocurre con la variable dependiente (tabla 8 ).

\begin{tabular}{|c|c|c|c|c|c|c|}
\hline \multicolumn{7}{|c|}{ Coefficients $^{\mathrm{a}}$} \\
\hline \multirow[b]{2}{*}{ Mod } & & \multicolumn{2}{|c|}{ Unstandardized Coefficients } & \multirow{2}{*}{$\begin{array}{c}\text { Standardized } \\
\text { Coefficients } \\
\text { Beta }\end{array}$} & \multirow[b]{2}{*}{$t$} & \multirow[b]{2}{*}{ Sig. } \\
\hline & & $B$ & Std. Error & & & \\
\hline \multirow[t]{4}{*}{1} & (Constant) & 3,774 &, 321 & & 11,755 &, 000 \\
\hline & AUTOESTIMA & -265 &, 058 & -165 & $-4,546$ &, 000 \\
\hline & CLIMAFAMILIAR &, 212 &, 062 &, 118 & 3,401 &, 001 \\
\hline & RIESGOSUICIDA & -182 &, 038 & -176 & $-4,795$ &, 000 \\
\hline
\end{tabular}

a. Dependent Variable: INDICEGLOBALDEACOSO

b. Predictors: (Constant), CLIMAFAMILIAR, AUTOESTIMA, RIESGOSUICIDA

Tabla 8. Análisis de los coeficientes de las variables: El clima familiar, autoestima, riesgo suicida y el indice global de acoso

La cuarta prueba de hipótesis de esta misma muestra dio $p=.000$, indicando que existe relación lineal significativa. Por lo tanto, se rechazó la hipótesis nula y se concluyó que la depresión, ansiedad, riesgo suicida y la intensidad de acoso están relacionados (tabla 9).

\begin{tabular}{|c|c|c|c|c|c|c|}
\hline \multicolumn{7}{|c|}{ ANOVA ${ }^{a}$} \\
\hline \multicolumn{2}{|c|}{ Model } & $\begin{array}{l}\text { Sum of } \\
\text { Squares }\end{array}$ & df & Mean Square & $\mathrm{F}$ & Sig. \\
\hline \multirow[t]{3}{*}{1} & Regression & 67,962 & 3 & 22,654 & 21,018 & $.000^{\mathrm{b}}$ \\
\hline & Residual & 877,355 & 814 & 1,078 & & \\
\hline & Total & 945,317 & 817 & & & \\
\hline
\end{tabular}

a. Dependent Variable: INTENSIDADDEACOSO

b. Predictors: (Constant), DEPRESION, ANSIEDAD, RIESGOSUICIDA

Tabla 9. Análisis de las variables: La depresión, ansiedad, riesgo suicida y la intensidad de acoso

De acuerdo con el nivel crítico asociado a cada prueba $t$, se concluyó que de las 3 variables utilizadas, 2 poseen coeficientes significativos $(p<$ $0,05)$. Por tanto, contribuyen a explicar lo que ocurre con la variable dependiente, excepto las variables riesgo suicida $(p=.181)$ y depresión $(p=$ .894), como se muestra en la tabla 10 .

\begin{tabular}{|c|c|c|c|c|c|c|}
\hline \multicolumn{7}{|c|}{ Coefficients $^{\mathrm{a}}$} \\
\hline \multirow[b]{2}{*}{ Model } & & \multicolumn{2}{|c|}{ Unstandardized Coefficients } & \multirow{2}{*}{$\begin{array}{c}\text { Standardized } \\
\text { Coefficients } \\
\text { Beta }\end{array}$} & \multirow[b]{2}{*}{$t$} & \multirow[b]{2}{*}{ Sig. } \\
\hline & & B & Std. Error & & & \\
\hline 1 & (Constant) & 1,487 &, 308 & & 4,833 &, 000 \\
\hline & RIESGOSUICIDA &,- 078 &, 058 &,- 090 & $-1,340$ & ,181 \\
\hline & ANSIEDAD & .400 &, 053 & .256 & 7,589 &, 000 \\
\hline & DEPRESION &,- 009 &, 070 &,- 009 &,- 133 &, 894 \\
\hline
\end{tabular}

a. Dependent Variable: INTENSIDADDEACOSO

b. Predictors: (Constant), DEPRESION, ANSIEDAD, RIESGOSUICIDA

Tabla 10. Análisis de los coeficientes de las variables: La depresión, ansiedad, riesgo suicida y la intensidad de acoso

La quinta prueba de hipótesis de esta misma muestra dio $p=.000$, indicando que existe relación lineal significativa. Por lo tanto, se rechazó la hipótesis nula y se concluyó que la autoestima, clima familiar, autoconcepto y la intensidad de acoso están relacionados (tabla 11).

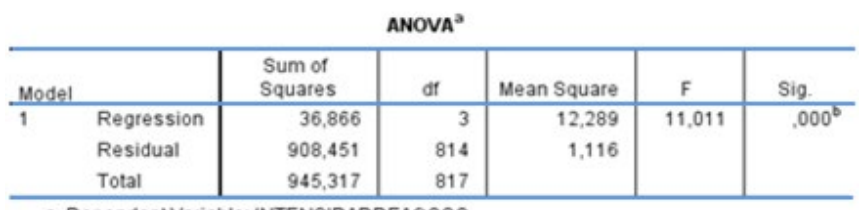

a. Dependent Variable: INTENSIDADDEACOSO

b. Predictors: (Constant), AUTOESTIMA CLIMAFAMILAR AUTOCONCEPTO,

Tabla 11. Análisis de las variables: La Autoestima, clima familiar, autoconcepto y la intensidad de acoso

De acuerdo con el nivel crítico asociado a cada prueba t, se concluyó que las 3 variables utilizadas poseen coeficientes significativos $(p<.05)$. Por tanto, contribuyen a explicar lo que ocurre con la variable dependiente (tabla 12).

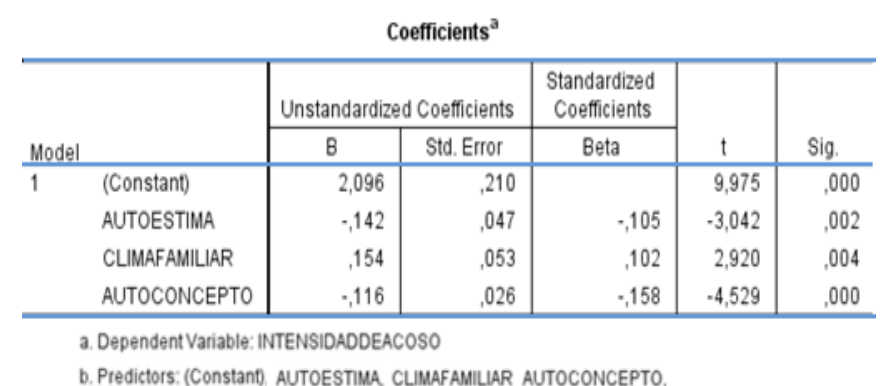

Tabla 12. Análisis de los coeficientes de las variables: La autoestima, clima familiar, autoconcepto y la intensidad de acoso

La sexta prueba de hipótesis de esta misma muestra se dio $p=.000$, indicando que existe relación lineal significativa. Por lo tanto, se rechazó la hipótesis nula y se concluyó que el clima familiar, autoestima, riesgo suicida e intensidad de acoso están relacionados (tabla 13). 
Modelo explicativo de factores psicológicos y el bullying: Primer proyecto Semillero de Investigación de la

Direccion Universitaria de Educación a Distancia de la Universidad Alas Peruanas

\begin{tabular}{ll|r|r|r|r|r}
\multicolumn{7}{c}{ ANOVA $^{\text {a }}$} \\
\hline \multicolumn{1}{l|}{} & \multicolumn{1}{c}{$\begin{array}{c}\text { Sum of } \\
\text { Model }\end{array}$} & \multicolumn{1}{c|}{ df } & Mean Square & F & \multicolumn{1}{c}{ Sig. } \\
\hline 1 & Regression & 24,647 & 3 & 8,216 & 7,264 &, $000^{5}$ \\
& Residual & 920,670 & 814 & 1,131 & & \\
\hline & Total & 945,317 & 817 & & & \\
\hline
\end{tabular}

a. Dependent Variable: INTENSIDADDEACOSO

b. Predictors: (Constant), CLIMAFAMILIAR, AUTOESTIMA RIESGOSUICIDA

Tabla 13. Análisis de las variables: El clima familiar, Autoestima, riesgo suicida y la intensidad de acoso

De acuerdo con el nivel crítico asociado a cada prueba $t$, se concluye que de las 3 variables utilizadas, 2 poseen coeficientes significativos $(p<.05)$. Por tanto, contribuyen a explicar lo que ocurre con la variable dependiente, excepto el clima familiar $(p=.094)$, según se observa en la tabla 14 .

\begin{tabular}{|c|c|c|c|c|c|c|}
\hline \multicolumn{7}{|c|}{ Coefficients $^{a}$} \\
\hline \multirow[b]{2}{*}{ Model } & & \multicolumn{2}{|c|}{ Unstandardized Coefficients } & \multirow{2}{*}{$\begin{array}{c}\text { Standardized } \\
\text { Coefficients }\end{array}$} & \multirow[b]{2}{*}{$t$} & \multirow[b]{2}{*}{ Sig. } \\
\hline & & $B$ & Std. Error & & & \\
\hline 1 & (Constant) & 2,441 &, 275 & & 8,880 & .000 \\
\hline & AUTOESTIMA &,- 185 &, 050 &,- 136 & $-3,704$ &, 000 \\
\hline & CLIMAFAMILIAR & .089 & .053 & .059 & 1,678 &, 094 \\
\hline & RIESGOSUICIDA &,- 100 & .033 &,- 115 & $-3,072$ &, 002 \\
\hline
\end{tabular}

Tabla 14. Análisis de los coeficientes de las variables: El clima familiar, autoestima, riesgo suicida y la intensidad de acoso

Prueba de hipótesis para los datos de 5 to grado.

En la primera prueba de hipótesis de los estudiantes del 5 to grado de primaria de las instituciones educativas de San Juan de Lurigancho (Lima), Cerro Azul (Arequipa) y El Porvenir (Trujillo) se obtuvo el valor del nivel crítico $(p=.000)$, que indica una relación lineal significativa. Por lo tanto, se rechazó la hipótesis nula y se concluyó que la depresión, ansiedad, riesgo suicida y el indice global de acoso están relacionados (tabla 15).

\begin{tabular}{|c|c|c|c|c|c|c|}
\hline \multicolumn{7}{|c|}{ ANOVA $^{3}$} \\
\hline \multicolumn{2}{|c|}{ Model } & $\begin{array}{l}\text { Sum of } \\
\text { Squares }\end{array}$ & df & Mean Square & $\mathrm{F}$ & Sig. \\
\hline \multirow[t]{3}{*}{1} & Regression & 51,460 & 3 & 17,153 & 11,921 &, $000^{\circ}$ \\
\hline & Residual & 1306,575 & 908 & 1,439 & & \\
\hline & Total & 1358,035 & 911 & & & \\
\hline
\end{tabular}

a. Dependent Variable: INDICEGLOBALDEACOSO

b. Predictors: (Constant), DEPRESION, ANSIEDAD RIESGOSUICIDA.

Tabla 15. Análisis de variables: La depresión, ansiedad, riesgo suicida y el indice global de acoso

De acuerdo con el nivel crítico asociado a cada prueba $t$, se concluyó que de las 3 variables utilizadas, 2 poseen coeficientes significativos $(p$ $<.05)$. Por tanto, contribuyen a explicar lo que ocurre con la variable dependiente, excepto el riesgo suicida $(p=.307)$, tal como se muestra en la tabla 16.

Coefficients $^{2}$

\begin{tabular}{|c|c|c|c|c|c|c|}
\hline \multirow[b]{2}{*}{ Mode } & & \multicolumn{2}{|c|}{ Unstandardized Coefficients } & \multirow{2}{*}{$\begin{array}{c}\begin{array}{c}\text { Standardized } \\
\text { Coefficients }\end{array} \\
\text { Beta } \\
\end{array}$} & \multirow[b]{2}{*}{$t$} & \multirow[b]{2}{*}{ Sig. } \\
\hline & & $B$ & Std. Error & & & \\
\hline \multirow[t]{4}{*}{1} & (Constant) & 1,667 &, 343 & & 4,856 & .000 \\
\hline & DEPRESION & .843 & .231 & 119 & 3,644 & .000 \\
\hline & ANSIEDAD & .235 & .056 &, 137 & 4,189 & .000 \\
\hline & RIESGOSUICIDA & .063 &, 061 & .033 & 1,022 &, 307 \\
\hline
\end{tabular}

a. Dependent Variable: INDICEGLOBALDEACOSO

b. Predictors: (Constant), DEPRESION, ANSIEDAD RIESGOSUICIDA.

Tabla 16. Análisis de los coeficientes de las variables: La depresión, ansiedad, riesgo suicida y el indice global de acoso

La segunda prueba de hipótesis de los estudiantes del 5to. grado de primaria dio $p=.197$, indicando que no existe relación lineal significativa. Por lo tanto, no se rechazó la hipótesis nula y se concluyó que la autoestima, clima familiar, autoconcepto y el indice global de acoso no están relacionados (tabla 17 ).

\begin{tabular}{ll|r|r|r|r|c}
\multicolumn{1}{c}{ ANOVA $^{\text {a }}$} \\
\hline Model & & \multicolumn{1}{c}{$\begin{array}{c}\text { Sum of } \\
\text { Squares }\end{array}$} & \multicolumn{1}{c|}{ df } & Mean Square & F & Sig. \\
\hline 1 & Regression & 6,829 & 3 & 2,276 & 1,562 &, $197^{\text {b }}$ \\
& Residual & 1322,956 & 908 & 1,457 & & \\
& Total & 1329,785 & 911 & & & \\
\hline
\end{tabular}

a. Dependent Variable: INDICEGLOBALDEACOSO

b. Predictors: (Constant), AUTOESTIMA, CLIMAFAMILIAR, AUTOCONCEPTO

Tabla 17. Análisis de Variables: La autoestima, clima familiar, autoconcepto y el indice global de acoso

De acuerdo con el nivel crítico asociado a cada prueba $t$, se concluyó que de las 3 variables utilizadas ninguna posee coeficientes significativos $(p>.05)$. En consecuencia ninguna contribuye a explicar de forma significativa lo que ocurre con la variable dependiente (tabla 18).

\begin{tabular}{|c|c|c|c|c|c|c|}
\hline \multicolumn{7}{|c|}{ Coefficients ${ }^{\mathrm{a}}$} \\
\hline \multirow[b]{2}{*}{ Model } & & \multicolumn{2}{|c|}{ Unstandardized Coefficients } & \multirow{2}{*}{$\begin{array}{c}\text { Standardized } \\
\text { Coefficients }\end{array}$} & \multirow[b]{2}{*}{$t$} & \multirow[b]{2}{*}{ Sig. } \\
\hline & & B & Std. Error & & & \\
\hline 1 & (Constant) & 3,726 & .231 & & 16,158 &, 000 \\
\hline & AUTOESTIMA & -074 & .061 &,- 041 & $-1,213$ & .226 \\
\hline & CLIMAFAMILIAR &,- 082 & .052 &,- 052 & $-1,575$ &, 116 \\
\hline & AUTOCONCEPTO &,- 014 &, 029 &,- 016 &,- 471 &, 638 \\
\hline
\end{tabular}

a. Dependent Variable: INDICEGLOBALDEACOSO

b. Predictors: (Constant), AUTOESTIMA, CLIMAFAMILIAR, AUTOCONCEPTO

Tabla 18. Análisis de coeficientes de variables: La autoestima, clima familiar, autoconcepto y el indice global de acoso

La tercera prueba de hipótesis de esta misma muestra dio $p=.003$, indicando que existe rela- 
ción lineal significativa. Por lo tanto, se rechazó la hipótesis nula y se concluyó que el clima familiar, autoestima, riesgo suicida y la intensidad de acoso están relacionados (tabla 19).

\begin{tabular}{|c|c|c|c|c|c|c|}
\hline \multicolumn{7}{|c|}{ ANOVA $^{a}$} \\
\hline Model & & $\begin{array}{l}\text { Sum of } \\
\text { Squares }\end{array}$ & df & Mean Square & $\mathrm{F}$ & Sig. \\
\hline \multirow[t]{3}{*}{1} & Regression & 16,150 & 3 & 5,383 & 4,731 &, $003^{b}$ \\
\hline & Residual & 1033,296 & 908 & 1,138 & & \\
\hline & Total & 1049,446 & 911 & & & \\
\hline
\end{tabular}

a. Dependent Variable: INDICEGLOBALDEACOSO

b. Predictors: (Constant), CLIMAFAMILIAR, AUTOESTIMA, RIESGOSUICIDA

Tabla 19. Análisis de las variables: El clima familiar, autoestima, riesgo suicida y el indice global de acoso

De acuerdo con el nivel crítico asociado a cada prueba $t$, se concluyó que de las 3 variables utilizadas, 1 posee coeficiente significativo $(p<.05)$. Por tanto, contribuye a explicar lo que ocurre con la variable dependiente, excepto riesgo suicida ( $p$ $=.601)$ y autoestima $(p=.087)$, según se observa en la tabla 20.

\begin{tabular}{|c|c|c|c|c|c|c|}
\hline \multicolumn{7}{|c|}{ Coefficients $^{a}$} \\
\hline \multirow[b]{2}{*}{ Model } & & \multicolumn{2}{|c|}{ Unstandardized Coefficients } & \multirow{2}{*}{$\begin{array}{c}\begin{array}{c}\text { Standardized } \\
\text { Coefficients }\end{array} \\
\text { Beta }\end{array}$} & \multirow[b]{2}{*}{$t$} & \multirow[b]{2}{*}{ Sig. } \\
\hline & & $B$ & Std. Error & & & \\
\hline \multirow[t]{4}{*}{1} & (Constant) & 2,736 &, 304 & & 8,998 &, 000 \\
\hline & CLIMAFAMILIAR &,- 146 &, 046 & -104 & $-3,152$ &, 002 \\
\hline & RIESGOSUICIDA &,- 034 &, 065 &,- 017 &,- 523 & 601 \\
\hline & AUTOESTIMA &,- 091 & .053 &,- 057 & $-1,715$ &, 087 \\
\hline
\end{tabular}

a. Dependent Variable: INDICEGLOBALDEACOSO

b. Predictors: (Constant), CLIMAFAMILIAR, AUTOESTIMA, RIESGOSUICIDA

Tabla 20. Análisis de los coeficientes de las variables: clima familiar, autoestima, riesgo suicida y el indice global de acoso

La cuarta prueba de hipótesis de esta misma muestra dio $p=.000$, indicando que existe relación lineal significativa. Por lo tanto, se rechazó la hipótesis nula y se concluyó que la depresión, ansiedad, riesgo suicida e intensidad de acoso no están relacionados (tabla 21).

\begin{tabular}{|c|c|c|c|c|c|c|}
\hline \multicolumn{7}{|c|}{ ANOVA $^{a}$} \\
\hline Model & & $\begin{array}{l}\text { Sum of } \\
\text { Squares }\end{array}$ & df & Mean Square & $\mathrm{F}$ & Sig. \\
\hline \multirow[t]{3}{*}{1} & Regression & 42,447 & 3 & 14,149 & 12,758 &, $000^{\mathrm{b}}$ \\
\hline & Residual & 1007,000 & 908 & 1,109 & & \\
\hline & Total & 1049,446 & 911 & & & \\
\hline
\end{tabular}

a. Dependent Variable: INTENSIDADDEACOSO

b. Predictors: (Constant), DEPRESION ANSIEDAD, RIESGOSUICIDA.

Tabla 21. Análisis de variables; La depresión, ansiedad, riesgo suicida y la intensidad de acoso

De acuerdo con el nivel crítico asociado a cada prueba $t$, se concluyó que de las 3 variables utilizadas, 2 poseen coeficientes significativos $(p<.05)$.
Por tanto, contribuyen a explicar lo que ocurre con la variable dependiente, excepto el riesgo suicida $(p=.402)$, según se muestra en la tabla 22 .

\begin{tabular}{|c|c|c|c|c|c|c|}
\hline \multicolumn{7}{|c|}{ Coefficients $^{a}$} \\
\hline & & \multicolumn{2}{|c|}{ Unstandardized Coefficients } & \multirow{2}{*}{$\begin{array}{c}\text { Standardized } \\
\text { Coefficients }\end{array}$} & \multirow[b]{2}{*}{$\mathrm{t}$} & \multirow[b]{2}{*}{ Sig. } \\
\hline \multicolumn{2}{|c|}{ Model } & B & Std. Error & & & \\
\hline \multirow[t]{4}{*}{1} & (Constant) & ,929 & ,322 & & 2,889 & ,004 \\
\hline & RIESGOSUICIDA &,- 053 & .064 &,- 027 &,- 839 & .402 \\
\hline & DEPRESION & 869 & 186 & 153 & 4,677 & ,000 \\
\hline & ANSIEDAD & 161 & ,049 & 108 & 3,295 & ,001 \\
\hline
\end{tabular}

Tabla 22. Análisis de coeficientes de las variables: La depresión, ansiedad, riesgo suicida y el indice global de acoso

La quinta prueba de hipótesis de esta misma muestra dio $p=.001$, indicando que existe relación lineal significativa. Por lo tanto, se rechazó la hipótesis nula y se concluyó que la autoestima, clima familiar, autoconcepto y la intensidad de acoso están relacionados (tabla 23).

\begin{tabular}{|c|c|c|c|c|c|c|}
\hline \multicolumn{7}{|c|}{ ANOVA $^{a}$} \\
\hline Model & & $\begin{array}{l}\text { Sum of } \\
\text { Squares }\end{array}$ & df & Mean Square & $\mathrm{F}$ & Sig. \\
\hline \multirow[t]{3}{*}{1} & Regression & 19,101 & 3 & 6,367 & 5,611 & $.001^{\mathrm{b}}$ \\
\hline & Residual & 1030,346 & 908 & 1,135 & & \\
\hline & Total & 1049,446 & 911 & & & \\
\hline
\end{tabular}

Tabla 23. Análisis de variables: La autoestima, clima familiar, autoconcepto y la intensidad de acoso

De acuerdo con el nivel crítico asociado a cada prueba t, se concluyó que de las 3 variables utilizadas, 1 posee coeficiente significativo $(p<.05)$. Por tanto, contribuye a explicar lo que ocurre con la variable dependiente, excepto autoconcepto $(p$ $=.090)$ y autoestima $(p=.163)$, según se aprecia en la tabla 24.

\begin{tabular}{|c|c|c|c|c|c|c|}
\hline \multicolumn{7}{|c|}{ Coefficients $^{a}$} \\
\hline \multirow[b]{2}{*}{ Model } & & \multicolumn{2}{|c|}{ Unstandardized Coefficients } & \multirow{2}{*}{$\begin{array}{c}\text { Standardized } \\
\text { Coefficients } \\
\text { Beta }\end{array}$} & \multirow[b]{2}{*}{$t$} & \multirow[b]{2}{*}{ Sig. } \\
\hline & & $B$ & Std. Error & & & \\
\hline 1 & (Constant) & 2,703 & .203 & & 13,283 & .000 \\
\hline & AUTOCONCEPTO & -.043 & .025 & -.057 & $-1,696$ & .090 \\
\hline & AUTOESTIMA & -.075 & .054 & -.047 & $-1,396$ & 163 \\
\hline & CLIMAFAMILIAR &,- 148 & .046 & -106 & $-3,211$ &, 001 \\
\hline
\end{tabular}

Tabla 24. Análisis de coeficientes de las variables: La autoestima, clima familiar, autoconcepto y la intensidad de acoso

La sexta prueba de hipótesis de esta misma muestra dio $p=.003$, indicando que existe relación lineal significativa. Por lo tanto, se rechazó la hi- 
Modelo explicativo de factores psicológicos y el bullying: Primer proyecto Semillero de Investigación de la Direccion Universitaria de Educación a Distancia de la Universidad Alas Peruanas

pótesis nula y se concluyó que el clima familiar, autoestima, riesgo suicida y la intensidad de acoso están relacionados (tabla 25).

\begin{tabular}{|c|c|c|c|c|c|c|}
\hline \multicolumn{7}{|c|}{ ANOVA $^{a}$} \\
\hline \multicolumn{2}{|l|}{ Model } & $\begin{array}{l}\text { Sum of } \\
\text { Squares }\end{array}$ & df & Mean Square & $\mathrm{F}$ & Sig. \\
\hline \multirow[t]{3}{*}{1} & Regression & 16,150 & 3 & 5,383 & 4,731 &, $003^{b}$ \\
\hline & Residual & 1033,296 & 908 & 1,138 & & \\
\hline & Total & 1049,446 & 911 & & & \\
\hline
\end{tabular}

a. Dependent Variable: INTENSIDADDEACOSO

b. Predictors: (Constant), CLIMAFAMILIAR, AUTOESTIMA RIESGOSUICIDA.

Tabla 25. Análisis de variables: El clima familiar, Autoestima, riesgo suicida y la intensidad de acoso

De acuerdo con el nivel crítico asociado a cada prueba t, se concluyó que de las 3 variables utilizadas, 1 posee coeficiente significativo $(\mathrm{p}<.05)$. Por tanto, contribuye a explicar lo que ocurre con la variable dependiente, excepto autoestima $(p=$ $.087)$ y riesgo suicida $(p=.601)$, tal como se observa en la tabla 26.

\begin{tabular}{|c|c|c|c|c|c|c|}
\hline \multicolumn{7}{|c|}{ Coefficients ${ }^{\mathrm{a}}$} \\
\hline & & \multicolumn{2}{|c|}{ Unstandardized Coefficients } & \multirow{2}{*}{$\begin{array}{c}\text { Standardized } \\
\text { Coefficients }\end{array}$} & \multirow[b]{2}{*}{$t$} & \multirow[b]{2}{*}{ Sig. } \\
\hline \multicolumn{2}{|c|}{ Model } & B & Std. Error & & & \\
\hline 1 & (Constant) & 2,736 & ,304 & & 8,998 & 000 \\
\hline & AUTOESTIMA &,- 091 & .053 &,- 057 & $-1,715$ & 087 \\
\hline & CLIMAFAMILIAR &,- 146 & .046 &,- 104 & $-3,152$ &, 002 \\
\hline & RIESGOSUICIDA &,- 034 &, 065 &,- 017 &,- 523 & 601 \\
\hline
\end{tabular}

Tabla 26. Análisis de coeficientes de las variables: El clima familiar, autoestima, riesgo suicida y la intensidad de acoso

\section{Discusión y Conclusiones}

En su teoría, Dan Olwes (1978) explica que aquellos estudiantes que sufren de bullying o acoso escolar tienden a sufrir de depresión, ansiedad y, en algunos casos, cuando el acoso es incesante, llegan a suicidarse o intentan hacerlo. Resultados similares obtuvo Sanchez-Sosa et al. (2010), que comprobó una relación directa entre la ideación suicida, la sintomatología depresiva y la victimización escolar. Estos aspectos coinciden con los resultados obtenidos en esta investigación, pues están relacionados la depresión, ansiedad, riesgo suicida y bullying (indice global de acoso) en los estudiantes de cuarto y quinto grado, siendo la ansiedad $(p=.000)$ la variable que explica mejor la incidencia en el Bullying (indice global de acoso) en ambos grados, seguido de la depresión $(p=$
.015 , en 4 to grado y $p=.000$ en 5 to grado). Esto coincide con el estudio realizado por Bowes et al. (2015), quien demostró que en aquellas personas que habían sufrido de bullying a los 13 años, el $14.8 \%$ sufre depresión a los 18 años y el $10.1 \%$ tuvo un episodio depresivo que duro más de dos años.

Según Moos (Casullo, s.f.), un buen clima familiar brinda a los estudiantes una mayor seguridad, autonomía e influye en su autoestima y autoconcepto. Les hace más sociables y favorece el desarrollo adecuado de dimensiones relacionadas. Se considera el grado de comunicación y libre expresión dentro de la familia la dimensión de desarrollo dentro de la cual es la importancia que brinda la familia al desarrollo personal y la dimensión de estabilidad compuesta por la estructura, la organización de la familia y el grado de control que ejercen los miembros de la familia. En el presente estudio, solo para el caso de los estudiantes del cuarto grado se demostró que existe una relación entre estas variables $(p=.000)$, con mayor influencia en clima familiar y bullying. Tal como menciona Muro (2010), la dinámica familiar en el acoso escolar se caracteriza por la presencia de desestructuración familiar: padres hostiles o familias con historia de violencia, existiendo una relación positiva en la formación de la persona con este perfil. A diferencia del quinto grado, donde no existe relación, es decir, el clima familiar no es un factor influyente en el bullying (índice global de acoso). Estos resultados coinciden con los hallazgos de Estela (2013), quien no encontró correlación entre clima social familiar y la conducta agresiva. También es relevante mencionar que entre el bullying (índice de acoso) hay una relación con autoestima, riesgo suicida, lo cual es preocupante porque aquellos estudiantes que obtuvieron puntajes altos han tenido ya más de un intento suicida, sin que sus familiares tengan conocimiento y perciban su suicidio como algo normal. Es en el caso de los estudiantes de 4to grado donde se obtuvo resultados similares a los obtenidos por Cano, Gutiérrez y Nizama (2009), quienes, en su investigación sobre tendencia a la violencia e ideación suicida en adolescentes, encontraron alta prevalencia de ideación suicida y haber pensado en quitarse la vida. En una muestra de 530 participantes, el $31.3 \%$ ha deseado morir, 
$21.1 \%$ pensó en quitarse la vida y el $18.1 \%$ pensó en quitarse la vida. A diferencia de los estudiantes de 5 to grado que solo se ha encontrado relación con autoestima, clima familiar y bullying (índice de acoso), no siendo influyente el riesgo suicida.

Ávila (2013) refiere que, según Coopersmith, la autoestima es la evaluación que el individuo hace y habitualmente mantiene con respecto de sí mismo y que lo demuestra sintiéndose significativo, valioso y exitoso, dando un juico personal de su valía. En los resultados de este estudio se puede concluir que la autoestima, clima familiar, autoconcepto y el bullying están relacionados $(p=$ $.002 ; p=.004 ; p=.000)$ en los estudiantes de cuarto grado de primaria. Según lo mencionado por Vexler (2012, septiembre 26), los victimarios y victimas presentan una débil estructura emocional, cuyo centro es su baja autoestima personal y social. Por su parte, León et al. (2011) refieren que hay efectos negativos en los estudiantes que sufren de acoso escolar, influyendo esto en un descenso de su autoestima o destruyéndola al extremo de llegar a estados depresivos o de permanente ansiedad. Martínez, (2009) en su investigación sobre "ajuste escolar, rechazo y violencia en adolescentes" refiere que los agresores o quienes no tienen problemas ni victimización presentaron niveles más elevados de autoestima social, mientras que las víctimas se sitúan en una posición baja en las cuatro dimensiones de autoestima (social, emocional, familiar y escolar), a diferencia de los estudiantes de 5 to grado, en quienes no se evidencia una relación entre el autoestima, clima familiar, autoconcepto y bullying (intensidad de acoso), no incidiendo estas variables en el bullying.

Con relación a la depresión, ansiedad, riesgo suicida y bullying (intensidad de acoso) en los estudiantes de cuarto y quinto grado, es la ansiedad la variable que explica mejor la incidencia del bullying $(p=.000$ y $p=.001)$ en ambos grados respectivamente. Este hallazgo coincide con lo mencionado por Ruíz y Lago (2005), quienes encontraron una prevalencia de $9-12 \%$, lo cual indica que es un problema de salud importante en la población infantojuvenil. En un artículo publicado en Perú 21 (noviembre 11, 2011) se enfatiza que los niños que han sufrido de maltrato infantil tienen mayor riesgo de desarrollar cualquier tipo de ansiedad, lo cual les puede llevar a la incapacidad. Por el contrario, no se ha encontrado una influencia de las variables riesgo suicida y depresión en el bullying en ninguno de los dos grados de primaria evaluados.

Los resultados obtenidos muestran una relación entre la autoestima, clima familiar, autoconcepto y bullying (intensidad de acoso) en ambos grados respectivamente $(p=.000 \mathrm{y} \mathrm{p}=.001)$ con mayor influencia en la variable clima familiar en ambos grados $(p=.000)$; solo autoconcepto y autoestima tienen una influencia en el bullying en 4to grado de primaria, no siendo significativas para el 5 to. Grado.

Aunque en diversas variables no se verificó una influencia en el bullying, en dos de los factores evaluados como son el índice de acoso global y la intensidad de acoso, el estudio permitió sugerir una serie de líneas de investigación en la temática del bullying, por constituir un problema latente a nivel mundial. Esto debe tenerse en cuenta para futuras investigaciones, como por ejemplo incluirlas en un estudio sobre el rendimiento académico de los estudiantes evaluados.

Además de las variables analizadas, seria relevante verificar si hay influencia del nivel socioeconómico, educación de los padres de familia, estilos de crianza, el género, la edad, así como evaluar el clima social escolar para verificar si tiene alguna influencia en el bullying. Dicho estudio puede realizarse con el modelo de ecuaciones estructurales.

Asimismo, debe evaluarse como una variable predictora las adicciones, sean consumo de sustancias, alcohol, tabaco, drogas ilícitas, juegos de azar o de tragamonedas y juegos online, debido a que en la evaluación en ciertas aulas los estudiantes estaban más pendientes de sus celulares. Se les veía manipulándolos constantemente, pese a la prohibición enunciada expresamente. Con relación al consumo de drogas, algunos de los estudiantes comentaron, por ejemplo, que ciertos compañeros fumaban a escondidas. 
Modelo explicativo de factores psicológicos y el bullying: Primer proyecto Semillero de Investigación de la Direccion Universitaria de Educación a Distancia de la Universidad Alas Peruanas

Agradecimiento: Muy especial a la Dirección Universitaria de Educación a Distancia de la Universidad Alas Peruanas por su confianza y financiamiento para implementar el semillero de Investigación de la División de Investigación y Extensión Científica Tecnológica, desde el ámbito de proyección y responsabilidad social. Igualmente a las estudiantes y docentes de la Escuela Académica de Psicología Humana por su participación y entrega en el desarrollo de cada una de las etapas realizadas del proyecto.

\section{REFERENCIAS BIBLIOGRÁFICAS}

Ávila, J. (2013). El acoso escolar en Educación Primaria en la provincia de Huelva. (Tesis doctoral inédita). Universidad de Huelva: Huelva.

Arroyave, P. (2012). Factores de vulnerabilidad y riesgo asociados al bullying. Revista CES Psicologia, 5 (1), 116-125.

Baltasar, D. (2014). Relación entre autoconcepto, ansiedad e inteligencia emocional: eficacia de un programa de intervención en estudiantes adolescentes. (Tesis doctoral inédita). Departamento de Psicología y Antropología, Universidad de Extremadura: España.

Bowes, L., Joinson, C., Wolke, D. y Lewis, G. (2015). Peer victimization during adolescence and its impact on depression in early adulthood: Prospective cohort study in the United Kingdom. doi: http://dx.doi.org/10.1136/bmj.h2469

Carreño, F. (2013). Investigación médica: el papel del estudiante de pregrado en la calidad científica de la universidad. Revista de los estudiantes de medicina de la Universidad Industrial de Santander. 26 (3) 57-60.

Cassullo, G. (s.f.). Algunas consideraciones acerca del concepto de Clima Social y su evaluación. Ficha de Catedra No.4, Facultad de Psicología. Universidad de Buenos Aires, Argentina.

Comité del Centro de Estudios para el adelanto de las mujeres y la Equidad de género. (2011). Estudio sobre violencia entre pares (bullying) en las escuelas de nivel básico en México. Folleto 1-4.

Corpas, E. (2009). La virtualización de los semilleros como alternativa de complementariedad. Revista médica de Risaralda. 15 (2) 53-60

Collell, J. y Escudé, C. (2006). El acoso escolar: un enfoque psicopatológico. Anuario de Psicologia Clínica, 2, 9-14. Recuperado de http://institucional.us.es/apcs/doc/APCS_2_ esp_9-14.pdf

Cano, P., Gutierrez, C. y Nizama, M. (2009). Tendencia a la violencia e ideación suicida en adolescentes escolares en una ciudad de la Amazonia Peruana. Rev Perú Exp. Salud pública, 26 (2), 175-181.
De la Ossa, J., Pérez, A., Patiño, R. y Montes, D. (2012). La investigación formativa como una necesidad en el pregrado. Revista Colombiana en Ciencia Animal. 4 (1), 1-3.

El Comercio. (2014, agosto 27). Más de mil casos de violencia escolar en Lima en el último año. Recuperado de http:// elcomercio.pe/lima/sucesos/mas-mil-casos-violencia-escolar-lima-ultimo-ano-noticia-1752801?ref=nota_lima\&ft=contenido

Estela, R. (2013). Clima familiar y su relación con la conducta agresiva de los estudiantes del VI ciclo de educación secundaria de la I.E. Francisco Bolognesi-Ugel 5 San Juan de Lurigancho. (Tesis de maestría inédita). Universidad César Vallejo: Lima.

Fernández, L. (2014, febrero 20). El bullying deja huellas en el $44 \%$ de los escolares limeños. Diario El Comercio. Recuperado de http://elcomercio.pe/lima/ciudad/bullying-deja-huellas-44-escolares-limenos-noticia-1711064

Gómez, C. (2014). Factores asociados a la violencia: revisión y posibilidades de abordaje. Revista Iberoamericana de Psicología: Ciencia y Tecnología, 7(1), 115-124.

Hernández, R. Fernández, C. y Baptista, P. (2014). Metodología de la investigación. México: Mg Graw Hill.

Hernández-Guzmán, L., Bermúdez-Ornelas, G., Spence, S. et al. (2009) Escala de Ansiedad para niños de Spence (SCAS). Revista Latinoamericana de Psicología, 42 (1) 1324.

La Republica (2014, septiembre 25). Cinco Distritos de Lima concentran casos de Bullying. Recuperado de http:// archivo.larepublica.pe/25-09-2014/cinco-distritos-de-lima-concentran-la-mayor-cantidad-de-casos-de-bullying

Leganes, E. (2013). Una propuesta de intervención para prevenir el acoso escolar desde una perspectiva sociogrupal. Psychology, Society \& education 2013, 5 (1) 21-40.

León, B., Gómez, T., Felipe, E., López, V. y García, A. (2011). Análisis del caso escolar en centros educativos de primaria en Extremadura. Revista desafíos y perspectivas actuales de la Psicologia en el mundo de la infancia, 1 (1) 561-572.

Loredo-Abdalá, A., Perea-Martínez, A. y López-Navarrete, G. (2008). "Bullying”: Acaso escolar. La violencia entre iguales. Problemática real en adolescentes. Acta pediátrica de México, 29 (4), 210-214.

Maritza, L., Aular de Durán, J. y Carruyo, J. (2013). Red de investigación estudiantil de la universidad del Zulia (REDIELUZ). Una política académica que enlaza la investigación y la tecnología. Enl@ce Revista Venezolana de Información, Tecnología y Conocimiento, 10 (3), 79-94.

Morales, O. Rincon, A. y Romero, J. (2005). Cómo enseñar a investigar en la universidad. Educere-Foro universitario, 9 (29) 217-224

Muro, M. (2010). Cólera y acoso escolar en un grupo de adolescentes de un colegio estatal en Lima metropolitana. (Tesis de licenciatura inédita) Pontificia Universidad Católica del Perú: Lima. 
Oliveros, M., Figueroa, L. Mayorga, G. Cano, B. Quispe, Y. y Barrientos, A. (2008). Violencia escolar (bullying) en colegios estatales de primaria en el Perú. Revista Peruana de Pediatría, 61 (4), 215-220.

Oliveros, M., Figueroa, L. Mayorga, G. Cano, B. Quispe, Y. y Barrientos, A. (2009). Intimidación en colegios estatales de secundaria del Perú. 62 (2), 68-78.

Perú 21(noviembre 11, 2011) Dos de cada diez niños sufren ansiedad. Recuperado de http://peru21.pe/noticia/1332031/dos-cada-diez-ninos-sufren-ansiedad

Pichicua, S. (2013, septiembre 10). Alarmante: al menos 20 adolescentes y niños se suicidaron. Diario Perú21. Recuperado de (http://peru21.pe/actualidad/alarmante-al-menos-20-ninos-y-adolescentes-se-suicidaron-2148560

RPP Noticias. (2014, agosto 30). El origen del bullying comienza en casa. Recuperado de http://www.rpp.com. pe/2014-08-30-el-origen-del-bullying-comienza-en-casanoticia_721097.html

Ruíz, A. y Lago, B. (2005). Trastornos de ansiedad en la infancia y en la adolescencia. Actualizaciones en Pediatría, 265-280. Recuperado de https://www.aepap.org/sites/default/files/ansiedad_0.pdf

Salgado, C. (2012). Revisión de las investigaciones acerca del Bullying: Desafíos para su estudio, 127-176. Recuperado de http://alfepsi.org/attachments/article/96/6\%20 Revisi\%C3\%B3n\%20de\%20las\%20investigaciones\%20 acerca $\% 20$ del $\% 20$ bullying $\% 20$ desaf $\%$ C3\%ADos $\% 20$ para\%20su\%20estudio\%20-\%20Salgado.pdf

Sánchez, M. (2014, febrero 2). Trujillo y Arequipa son las dos ciudades más violentas del país. Diario Perú21. Recuperado de http://peru21.pe/actualidad/trujillo-y-arequipa-son-dos-regiones-mas-violentas-pais-2168262

Sanchez-Sosa, J., Musitu, G., Villarreal-González, M. y Martínez, B. (2010). Ideación suicida en adolescentes: Un análisis psicosocial. Intervención psicosocial, 19 (3), 279287. DOI: $10.5093 /$ in2010v19n3a8

Vexler, I. (2012, septiembre 26). El bullying y la autoestima. Diario Correo. Recuperado de http://diariocorreo.pe/historico/el-bullying-y-la-autoestima-230383/http://www.uco. es/laecovi/ 